\title{
Risk for physical dependence in community-dwelling older adults: The role of fear of falling, falls and fall-related injuries
}

\author{
Catarina Pereira, ${ }^{1,2}$ (i) | Jorge Bravo, ${ }^{1,2}$ (I) | Armando Raimundo ${ }^{1,2}$ (i) | \\ Pablo Tomas-Carus $^{1,2}$ (D) | Felismina Mendes ${ }^{2,3}$ (D) | Fátima Baptista ${ }^{4}$ (D)
}

${ }^{1}$ Departamento de Desporto e Saúde, Escola de Ciências e Tecnologia, Universidade de Évora, Évora, Portugal

${ }^{2}$ Comprehensive Health Research Center (CHRC), Évora, Portugal

${ }^{3}$ Escola Superior de Enfermagem S. João de Deus, Universidade de Évora, Évora,

Portugal

${ }^{4}$ Exercise and Health Laboratory, Interdisciplinary Center for the Study of Human Performance, Faculty of Human Kinetics, Technical University of Lisbon, Lisbon, Portugal

Correspondence

Catarina Pereira, Departamento de Desporto e Saúde, Escola de Ciências e Tecnologia, Universidade de Évora; Colégio Luís António Verney, Rua Romão Ramalho 59, 7000-671 Évora, Portugal.

Email: clnp@uevora.pt

Funding information

This study was funded by the European Fund for Regional Development through Horizon 2020-Portugal 2020-Programa Operacional Regional do Alentejo (ALT2003-0145-FEDER-000007). The sponsors had no role in the design, analysis or preparation of this manuscript.

\begin{abstract}
Background: Falls and fall-related injuries along with fear of falling (FoF) seem to restrict activities of daily living (ADL), resulting in physical dependence. However, it is still unclear how falls and related injuries or FoF by themselves explain general and specific ADL dependence.
\end{abstract}

Objectives: To investigate the relationships between falls and related injuries, FoF and physical dependence on ADL in community-dwelling older adults, controlling for age, gender, physical activity and physical fitness as confounders.

Methods: This cross-sectional descriptive study assessed 588 community-dwelling older adults. Falls and fall-related injuries, ADL dependence on basic, instrumental and advanced activities, FoF, demographic characteristics and health conditions were assessed through a questionnaire. Physical activity was measured through the International Physical Activity Questionnaire. Physical fitness was assessed by the Senior Fitness Test and the Fullerton Advanced Balance Scale. Body composition was measured through bioimpedance.

Results: Severe injuries occurrence increased the likelihood of moderate and high physical dependence by 3 and 6 times, while FoF increased this likelihood by 3 and 7 times, respectively. Also, the occurrence of previous falls, resulting in severe injuries, increased the likelihood of dependence in two instrumental ADL (3 and 4 times), while FoF increased this likelihood in numerous basic, instrumental and advanced ADL (2-3 times). The FoF was shown to explain overall physical functioning dependence, by itself, representing a constraint on the performance of most basic, instrumental and advanced ADL.

Conclusion: The FoF showed to be a greater threat to ADL dependence than falls and related injuries. Assessment guidelines for older adults living in the community should include the FoF in clinical evaluation.

Implications for practice: Understand the isolated interplay of FoF and previous falls and injuries on $\mathrm{ADL}$ dependence among older adults allows healthcare professionals 
to perform more accurate clinical evaluations and develop more successful interventions to prevent further dependence.

\section{KEYWORDS}

accidental falls, activities of daily living, ageing, fear, independent living, physical fitness, practice guidelines

\section{INTRODUCTION}

Falls and fall-related injuries have been reported as a major factor restricting physical independence, becoming the main reason for the institutionalisation of older people (Choi \& Ko, 2015; Scheffer, Schuurmans, Van Dijk, Van Der Hooft, \& De Rooij, 2008). In addition to the physical consequences of falls, the psychological consequences, particularly the fear of falling (FoF), restrict the activities of daily living (ADL), resulting on dependence and loss of autonomy (Denkinger, Lukas, Nikolaus, \& Hauer, 2015; Hoang, Jullamate, Piphatvanitcha, \& Rosenberg, 2017; Ruthig, Chipperfield, Newall, Perry, \& Hall, 2007). FoF concern the degree of fear or confidence a person has in performing common ADL without falling (Tinetti, Mendes de Leon, Doucette, \& Baker, 1994; Tinetti, Richman, \& Powell, 1990) and was shown to be one of the most important factors in predicting future falls, even among those who never experienced a previous fall (Gómez, Wu, Auais, Vafaei, \& Zunzunegui, 2017). Several cross-sectional studies have identified a relationship between FoF and physical and mental performance, causing dependence on ADL (Scheffer et al., 2008).

Activities of daily living include basic activities of daily living (BADL) such as personal care, hygiene and getting around indoors; instrumental activities of daily living (IADL) encompassing transportation facilities, shopping, preparing meals and paying bills; and advanced activities of daily living (AADL) which comprises more demanding activities that enrich life, such as social, religious or leisure activities (Pereira, Baptista, \& Cruz-Ferreira, 2016; Rikli \& Jones, 1998, 2013). Besides the maintenance of BADL, which allows older people to remain living independently in the community, IADL and AADL are crucial for successful ageing, enabling enjoyment and quality of life (Bowling \& Iliffe, 2006; Rubio, Lazaro, \& SanchezSanchez, 2009). However, it is not clear in the literature how falls, fall-related injuries or FoF, by themselves, correlate with general and specific ADL dependence.

Several other factors associated with the ability to perform ADL were also recognised as risk factors for falls and fall-related injuries (Himes \& Reynolds, 2012; Pereira et al., 2016; Pereira, Baptista, \& Infante, 2014). Most of these are associated with the subject's intrinsic factors, such as age, health conditions, physical fitness, body composition, FoF and among frailer individuals, cognition status (Fujiwara et al., 2008; Lavedan et al., 2018). Habitual physical activity has also been identified as a key element in the ability to perform ADL and in the occurrence of falls and fall-related injuries (Paterson \& Warburton, 2010; Tinetti \& Kumar, 2010), particularly by the

\section{What does this research add to existing knowledge in gerontology?}

- This is the first study investigating the isolated role of falls, fall-related injuries and fear of fall (FoF) in physical dependence on activities of daily living (ADL).

- The FoF showed to be the greatest threat to ADL dependence, independently of previous falls and related injuries.

\section{What are the implications of this new knowledge for nursing care with older people?}

- Primary care nurses should assess FoF among community-dwelling older adults.

- Primary prevention education should focus on the importance of understanding the isolated interplay of FoF on ADL dependence among older adults.

\section{How could the findings be used to influence policy or practice or research or education?}

- Clinicians and researchers should consider including the FoF in clinical evaluation guidelines.

- The FoF evaluation might support geriatric program managers on the development of effective interventions targeting physical dependence risk reduction in older adults.

influence of physical activity and exercise on older adults' physical fitness and health (Chodzko-Zajko et al., 2009).

Despite previous similar approaches (Curcio, Gomez, \& ReyesOrtiz, 2009) to our knowledge, this is the first study investigating the role of falls, fall-related injuries and FoF in physical dependence among Portuguese older adults, particularly according to ADL categories (AADL vs. IADL vs. BADL), controlled for possible confounders. It was hypothesised that physical dependence might be related to FoF, previous falls and fall-related injuries. Thus, this study aimed to analyse the relationships between falls and related injuries, FoF and physical dependence, mainly according to each ADL category, this as regard age, gender, physical activity and physical fitness of community-dwelling older adults. An 
appropriated approach to these relationships would improve the understanding of the factors that can lead to physical dependence of older adults and, therefore, would help nurses and other healthcare professionals in the development of tailored prevention programs for ADL dependence.

\section{2 | METHODS}

\section{1 | Study design}

This study is an observational cross-sectional study.

\section{2 | Participants}

The population of the catchment area comprised communitydwelling Portuguese older adults. Participants were recruited by invitation and in response to leaflets and posters distributed in community settings (health centres, recreational, sports and cultural associations, universities for seniors, etc.). The minimum sample size representative of this population surveyed by the national census (INE, 2019) (adults $\geq 65$ years old: $N=10.276,617$ ) was calculated by the epidemiologic statistical OPENEPI software (Dean, Sullivan, \& Soe, 2014) as 385 (considering 95\% Cl). Six hundred and thirty-seven 637 older adults agreed to participate in the study. Participation required the absence of cognitive impairment (scoring $>24$ points in the Folstein Mini-Mental State Examination) (Folstein, Folstein, \& McHugh, 1975) and to be $\geq 65$ years old. Subjects who recently registered acute events resulting in a temporary dependence or change of physical fitness were excluded (e.g., myocardial infarction or fractures leading to immobilisation). Therefore, 49 respondents were excluded (including four volunteers who had a recent hip fracture). There remained 588 participants with $7.1 \pm 4.4$ years of school (464 women: $67.8 \pm 6.8$ years; 124 men: $69.6 \pm 6.5$ years), of whom $76 \%$ had exercised at least twice a week in the previous year. All participants were volunteers and provided informed consent. The study was approved by an institutional review board.

\section{3 | Outcome measures}

The measures were undertaken at the University of Évora, in the Technical University of Lisbon, and in community settings that have sports facilities. Assessments were performed by a specialised team of four raters trained in the protocols (graduated in sport sciences and experimented raters). Each test and questionnaire was always performed by the same rater, who was blind to the study's objectives. As many participants could not read or write because they had never attended school, a single interviewer completed the questionnaires through an interview for all participants. Each participant assessment took about an hour and a half. The intrarater reliability (with a one-week interval between test and retest performed on ten participants) was calculated using the bivariate correlation of Spearman or Pearson (Rose, Lucchese, \& Wiersma, 2006) and ranged from 0.722 to 0.999 .

\subsection{Physical function on activities of daily living}

Physical function was determined by responses to the 12 items on the Composite Physical Function (CPF) Scale (Rikli \& Jones, 1998), capable of assessing physical independence across a wide range of ADL (Rikli \& Jones, 2013). In this composite, items 1-3 concerns BADL, items 4-10 concerns IADL, and items 11 and 12 concerns AADL. Participants were asked to indicate whether they could do the activity (score 2), could do it with difficulty or with help (score 1) or could not perform the activity at all (score 0). The total CPF score ranges from 0 to 24 points. Participants were categorised as "high function" (score 24), "moderate function" (score 18 - 23) and "low function" (score < 18). In addition, as the participants were required to live independently in the community, each of the 12 itemised activities was also categorised dichotomously as follows: "independent" (can do: score 2) versus "dependent" (cannot do or can do with difficulty or help: score 0 or 1 , respectively).

\subsection{1 | Falls and fall-related injuries}

The occurrence of falls in the previous 12 months (no: 0; yes: 1) and its consequences (fall-related injuries) were exhaustively assessed by the interviewer using a questionnaire. Injury severity was classified as light (no injury, light scratches and oedema) or severe (serious abrasion, strained muscles, torn muscles, sprains, dislocations and fractures) (Pereira et al., 2014). Therefore, the occurrence of falls and injuries variable was expressed as follows: no falls; occurrence of at least one fall in previous years with no injury; occurrence of at least one fall with light injury; and occurrence of at least one fall with severe injury. This variable was also categorised as trichotomies (no falls or at least one fall with no injury; at least one fall with light injury; and at least one fall with severe injury) and as dichotomous (injury-: no falls or at least one fall without injury or with light injury; and injury+: designating the occurrence of at least one fall with severe injury) in order to facilitate clinical interpretation.

\subsection{2 | Fear of falling}

FoF was assessed by Falls Efficacy Scale (FES) used by Pluijm et al. (2006) and adapted from Tinetti et al. (1990). Participants were asked how concerned they felt about falling while performing each of the ten everyday activities listed in the FES. Each item was rated on a point scale from 0 (not concerned) to 3 (very concerned). The total score was the sum of the points obtained in each of the tests, ranging from 0 to 30 . In order to facilitate clinical interpretation, the 
FoF was classified into three FES categories, namely FES- (assumed as no fear): score $0-1$ versus FES \pm (assumed as some fear): score 2-3 versus FES+ (assumed as high fear): score $\geq 4$.

\subsection{3 | Physical fitness and body composition}

Lower and upper body strength (number of repetitions for $30 \mathrm{~s}$ ) and flexibility (cm), agility and dynamic balance (sec) and aerobic endurance $(\mathrm{m})$ and body mass index $\left(\mathrm{kg} / \mathrm{m}^{2}\right)$ were evaluated using the Senior Fitness Test battery (Rikli \& Jones, 2013). Multidimensional balance was evaluated by conducting the 10 tests of the Fullerton Advanced Balance (FAB) Scale (Rose et al., 2006). The final score, ranging from 0 to 40 points, was the sum of points obtained in each of these 10 tests, ranging from 0 (worst) to 4 (best). Standing height $(\mathrm{cm})$ was measured with a stadiometer (Secca 770, Hamburg, Germany) and weight (kg) using an electronic scale (Secca Bella 840, Hamburg, Germany). Fat body mass (\%) was evaluated by bioimpedance (Zaluska, Malecka, Mozul, \& Ksiazek, 2004) (HBF-306C), and lean body mass index $\left(\mathrm{kg} / \mathrm{m}^{2}\right)$ was calculated as (total body mass $[\mathrm{kg}]$ - fat body mass $[\mathrm{kg}]) /\left(\right.$ body height $\left.{ }^{2}\left[\mathrm{~m}^{2}\right]\right)$.

\subsection{4 | Physical activity}

Habitual physical activity was assessed using the short version of the International Physical Activity Questionnaire (IPAQ) (Craig et al., 2003). This questionnaire covers metabolic expenditure [metabolic equivalent of task [MET] - min/week) on walking (3.3 MET), moderate activity (4.0 MET) and vigorous activity (8.0 MET). Total metabolic expenditure (MET-min/week) was calculated by determining the time (min/day) and frequency (day/week) spent on each of these activities. Supervised exercise ( $\mathrm{hr} /$ week) was also assessed by means of questionnaire.

\subsubsection{Age, education level and health conditions}

Chronologic age, education level, and chronic diseases, physical impairments and age were assessed using a questionnaire. Participants listed a total of 24 chronic diseases. Physical impairments included involuntary loss of urine, frequent dizziness, foot problems, poor vision, hearing problems and occasional loss of balance (Pluijm et al., 2006). The presence or absence of each one of the listed chronic diseases or physical impairment was checked for each participant. The number of chronic diseases and physical impairments defined the variable "health conditions."

\section{5 | Statistical analysis}

An exploratory analysis was performed to characterise the data, to identify outliers and influential data, to test associations between the variables (using Pearson correlation and several univariate regression analysis) and to determine which cut-offs and categories should be considered in the studied variables, based on multinomial and binary logistic regression analysis $(p<.05)$.

Comparison of subjects' characteristics between groups according to their physical function level in CPF scale (high, moderate and low physical function) was performed by one-way ANOVA followed by the Bonferroni post hoc test to identify pairwise differences. In the case of no homoscedasticity, comparisons between groups were carried out by conducting the Kruskal-Wallis test, followed by multiple simultaneous examinations using the Mann-Whitney test. Comparisons of percentages (male/female; low/moderate/highly active; no fall/ fall-related light injury/fall-related severe injury) between groups were carried out using chi-square test or Fisher's test. Multinomial logistic regression was used to quantify the role of FoF, falls and fall-related injuries on physical function level (high, moderate and low physical function). Binary logistic regressions using the forward stepwise conditional method were used to quantify the role of FoF, falls, and fall-related injuries on dependence in each of the 12 activities reported in the CPF (dependent vs. independent). Therefore, adjustments were performed for potential confounders by multinomial or binary regression analysis as regards the other independent variables selected as significant to explain the dependent variable in each building model. Analysis performed with multiple simultaneous tests was carried out using the Bonferroni correction for $p$-value. Statistical analyses were performed using the sPSs software package (v. 21.0 for Windows; IBM), and statistical significance was set at $p \leq .05$.

\section{3 | RESULTS}

\subsection{Characterisation and exploratory results}

The sample characteristics analysis showed that the group of participants with low physical function was older and less healthy than the group with moderate physical function or with high physical function (Table 1). In general, these participants had worse body composition and physical fitness and were more afraid of falling, more likely to fall and to suffer injury and also less active. The group of participants with high physical function showed the best results for these variables $(p<.05)$.

Thirty-one per cent of the participants experienced at least one fall in the previous 12 months, totalling 336 falls; $9 \%$ of the participants experienced severe fall-related injuries (34 fractures, 12 dislocations, 19 sprains, 8 torn muscles, 5 strains and 16 serious abrasions), and $21 \%$ had no injuries or experienced light fall-related injuries (101 slight oedemas and 145 slight scratches). The exploratory analysis highlighted significant correlations between health conditions, physical activity, physical fitness, body composition and FoF, both for fallers and for non-fallers, in which a poor result on any one of the first variables was associated with a higher FoF $(p<.05)$. Moreover, univariate linear regression performed in the exploratory analysis showed that these variables explain FoF in a range from $4 \%$ (total 
TABLE 1 Characteristics of study participants by ADL category

\begin{tabular}{|c|c|c|c|c|c|}
\hline Variables & $\begin{array}{l}\text { a }(n=100) \\
\text { Low function }\end{array}$ & $\begin{array}{l}\mathrm{b}(n=331) \\
\text { Moderate function }\end{array}$ & $\begin{array}{l}c(n=157) \\
\text { High function }\end{array}$ & $p$ & Post hoc test \\
\hline CPF (points) & $15.0 \pm 2.4$ & $21.0 \pm 1.6$ & $24.0 \pm 0.0$ & $<.001$ & $\mathrm{a}<\mathrm{b}<\mathrm{c}$ \\
\hline Health conditions ( $n$ ) & $5.2 \pm 2.5$ & $3.4 \pm 2.1$ & $2.2 \pm 1.9$ & $<.001$ & $a<b<c$ \\
\hline Body mass index $\left(\mathrm{kg} / \mathrm{m}^{2}\right)$ & $29.4 \pm 5.0$ & $28.1 \pm 4.3$ & $28.2 \pm 4.3$ & .030 & $a>b$ \\
\hline Fat body mass (\%) & $42.9 \pm 4.5$ & $40.2 \pm 5.5$ & $36.2 \pm 6.6$ & $<.001$ & $a>b>c$ \\
\hline Lower body strength (rep) & $14.6 \pm 4.3$ & $16.4 \pm 4.5$ & $18.0 \pm 4.3$ & $<.001$ & $a<b<c$ \\
\hline Upper body strength (rep) & $15.2 \pm 3.6$ & $17.2 \pm 4.2$ & $19.2 \pm 5.0$ & $<.001$ & $a<b<c$ \\
\hline Lower body flexibility $(\mathrm{cm})$ & $-2.5 \pm 9.1$ & $-1.0 \pm 9.5$ & $-1.4 \pm 9.3$ & .384 & - \\
\hline Upper body flexibility $(\mathrm{cm})$ & $-14.0 \pm 10.5$ & $-8.9 \pm 10.8$ & $-7.9 \pm 10.3$ & $<.001$ & $\mathrm{a}<\mathrm{b}<\mathrm{c}$ \\
\hline Exercise (hr/week) & $1.8 \pm 1.2$ & $1.9 \pm 1.2$ & $2.3 \pm 1.3$ & .001 & $a, b<c$ \\
\hline Total PA (MET-min/week) & $2,436 \pm 1,807$ & $2,729 \pm 1,405$ & $3,298 \pm 1,956$ & $<.001$ & $a, b<c$ \\
\hline Walking (MET-min/week) & $544 \pm 489$ & $741 \pm 551$ & $825 \pm 545$ & $<.001$ & $a<b, c$ \\
\hline Moderate PA (MET-min/week) & $1,801 \pm 1,326$ & $1,885 \pm 1,172$ & $2,024 \pm 1,370$ & .342 & - \\
\hline Vigorous PA (MET-min/week) & $90 \pm 536$ & $103 \pm 407$ & $432 \pm 1,134$ & $<.001$ & $a, b<c$ \\
\hline Number of falls & $0.9 \pm 1.6$ & $0.6 \pm 1.3$ & $0.33 \pm 0.7$ & .002 & $a<b, c$ \\
\hline FoF score (point) & $5.6 \pm 5.5$ & $2.2 \pm 2.8$ & $0.9 \pm 1.7$ & $<.001$ & $\mathrm{a}<\mathrm{b}<\mathrm{c}$ \\
\hline \multicolumn{6}{|l|}{ Fall-related injuries } \\
\hline
\end{tabular}

Note: Data are mean $\pm S D$ or absolute frequency and percentage.

a, low function group; b, moderate function group; c, high function group.

Abbreviations: CPF, composite physical function; FoF, fear of falling; PA, physical activity.

physical activity $R^{2}=0.036$ ) to $18 \%$ (health conditions $R^{2}=0.178$ ), while the occurrence of falls and of severe injuries only explain FoF at $9 \%\left(R^{2}=0.091\right.$ and $R^{2}=0.089$, respectively, $\left.p<.05\right)$. Most important, this statistical technique showed that total FES score explained total CPF score on $34.8 \%\left(R^{2}=0.248, p<.001\right)$ and that falls occurrence (yes/no) explained total CPF score on $1.5 \%\left(R^{2}=0.015, p<.003\right)$.

Concerning physical independence, the percentage of participants who could do, could not do or do with difficulty or help each of the 12 itemised ADL in the CPF is illustrated in Figure 1. Over $95 \%$ of subjects had no difficulty performing BADL, and only $\sim 1.5 \%$ reported difficulty performing items 1 and 2. Regarding IADL, the percentage of dependent participants ranged from $4 \%$ in relation to less demanding activities to $59 \%$ for more demanding activities. Dependence in performing AADL was reported by $39 \%$ of participants for item 11 and by $59 \%$ of participants for item 12.

\subsection{General physical functioning main results}

Considering the three categories of physical functioning level, multinomial logistic regression was used to quantify the likelihoods of people to have moderate or low physical function due to the occurrence of falls with severe injury (Injury+) and due to FoF (some fear: FES \pm or high fear: FES+). Figure 2 shows these results, which were computed, taking into account the reference category "high physical function." Note that the occurrence of falls without injuries or with light injuries has not shown to significantly explain physical functioning level. The figure illustrates how subjects who had a severe fall-related injury were 2.5 times more likely to have moderate physical function (OR: $2.500,95 \% \mathrm{Cl}: 1.050-5.950)$ and 5.6 times more likely to have low physical function (OR: 5.548, 95\% Cl: 1.521-20.240) than subjects without severe injuries (who had not fallen, or had fallen but had experienced no injury, or had 
घan do $\quad$ Cannot do or can do with difficulty or help.

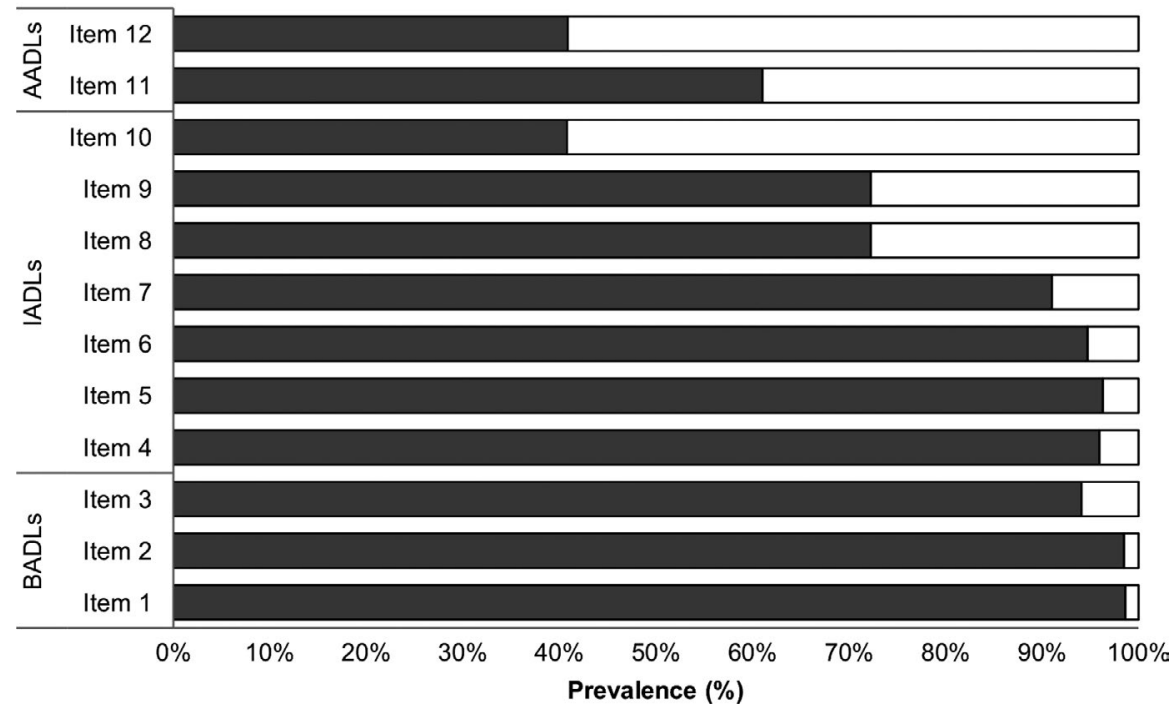

FIGURE 1 Prevalence of disability in the 12 activities of daily living evaluated according to the Composite Physical Function Scale. AADLs, advanced activities of daily living; IADLs, instrumental activities of daily living; BADLs, basic activities of daily living. Item 1, "Take care of own personal needs, such as dressing yourself"; Item 2, "Bathe yourself, using tub or shower"; Item 3, "Walk outside (1 or 2 blocks)"; Item 4, "Do light household chores, such as cooking, dusting, washing dishes, sweeping a walkway"; Item 5, "Climb up and down a flight of stairs"; Item 6, "Do own shopping and errands (walk approx 3-4 blks; 400 yds)"; Item 7, "Lift and carry 10 pounds (bag of groceries)"; Item 8, "Walk 1/2 mile (6-7 blocks)"; Item 9, "Walk 1 mile (12-14 blocks)"; Item 10, "Lift and carry 25 lb (medium to large suitcase)"; Item 11, "Do heavy household activities-like scrubbing floors, vacuuming, raking leaves"; Item 12, "Do strenuous activities-like hiking, digging in the garden, moving heavy objects, cycling, aerobic dance activities, strenuous calisthenics"

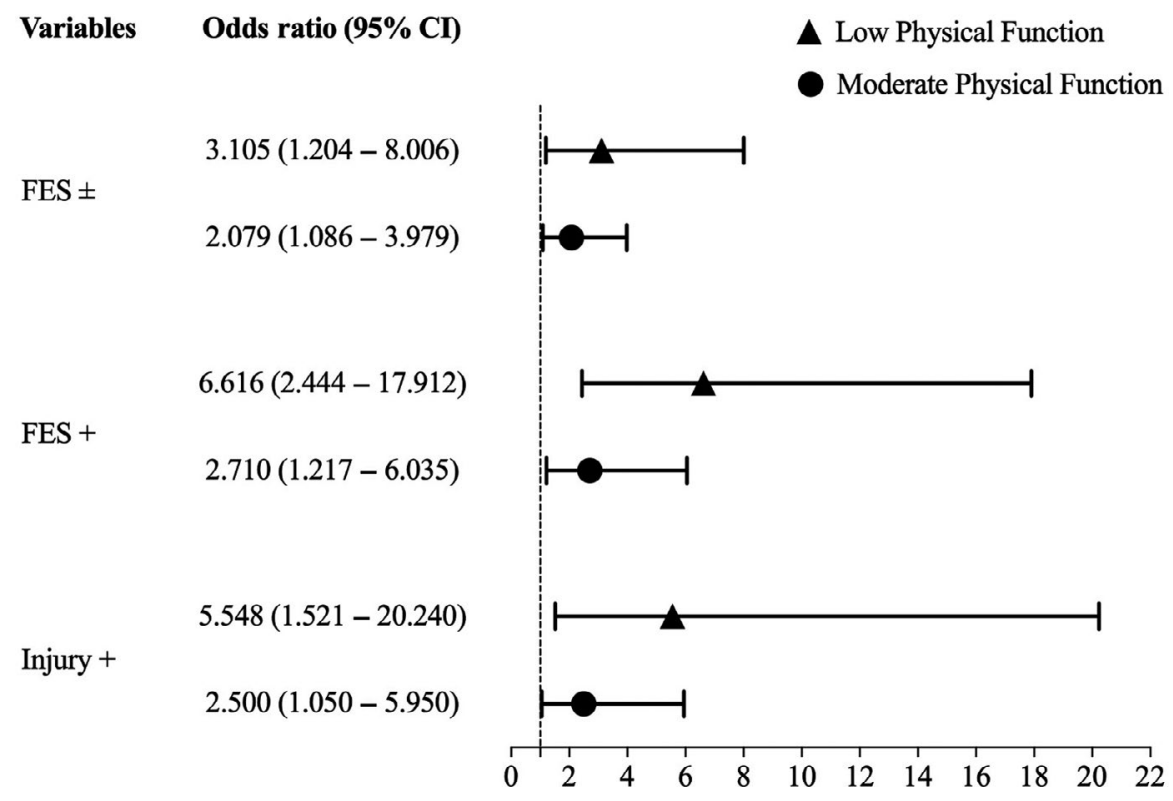

FIGURE 2 Odds ratio for physical functioning level associated with fallrelated injuries and fear of falling (results computed in opposition to high physical function category). The figure illustrates the likelihood of a person to have low or moderate physical function associated with the occurrence of severe fall-related injuries and fear of falling. The reference categories were as follows: no fall, or fall without injury or with light injury (Injury-) and with no fear of falling (FES-: score $0-1)$. $\mathrm{Cl}$, confidence interval fallen and had experienced light injuries). With an increase in FoF, in contrast to a person with no FoF (FES-), the likelihood of a person to have moderate physical function doubled or virtually tripled (OR: $2.079,95 \% \mathrm{Cl}: 1.086-3.979$ for FES \pm ; OR: $2.710,95 \% \mathrm{Cl}$ : 1.217-6.035 for FES+), being the likelihood of a person to have low physical function three or almost seven times higher (OR: 3.105, 95\% Cl: 1.204-8.006 for FES \pm ; OR: 6.616, 95\% Cl: 2.444-17.912 for FES+). In this analysis, values were adjusted for confounders.

\subsection{General physical functioning secondary results}

The analyses of secondary results (Table 2 ) showed that the selected confounders and the respective associated likelihood for moderate and low physical function computed by multinomial logistic regression were as follows: health conditions (moderate physical function OR: 1.172, 95\% Cl: 1.030-1.332; low physical function OR: $1.500,95 \% \mathrm{Cl}: 1.268-1.775)$, aerobic endurance (moderate physical 
function OR: 0.994, 95\% Cl: 0.991-0.998; low physical function OR: 0.989, 95\% Cl: 0.983-0.994), multidimensional balance (moderate physical function OR: $0.943,95 \% \mathrm{Cl}$ : 0.883-0.999; low physical function OR: $0.901,95 \% \mathrm{Cl}: 0.828-0.979)$ and lean body mass index (moderate physical function OR: $0.752,95 \% \mathrm{Cl}$ : 0.669-0.845; low physical function OR: $0.747,95 \% \mathrm{Cl}: 0.631-0.883)$. Physical activity variables, age and gender were not selected as significant predictors of physical functioning dependence by multinomial logistic regression modelling. Therefore, adjustment for previous results did not include these variables.

\section{4 | AADL, IADL and BADL dependence main results}

The risk of dependence in each AADL, IADL and BADL item due to falls and injuries and due to FoF was quantified by means of binary logistic regression. Figure 3 shows these main results. The figure illustrates how the occurrence of falls with severe injuries increased the likelihood of dependence by $\sim 3$ to 4 times in two ADL (OR: 2.665, 95\% Cl: 1.091-6.512, for item 9; OR: 3.777, 95\% Cl: $1.678-$ 8.501, for item 10). Reporting a FoF score of 2-3 (FES \pm ), in contrast to the FES- category (score $0-1$ ), increased the chance of dependence in item 9 (lift and carry $10 \mathrm{lb}$ ) by 2 times (OR: 2.195, 95\% Cl: 1.140-4.223), while reporting a FoF score $\geq 4$ (FES+) increased the likelihood of dependence in six ADL by 2-3 times (from OR: 1.994, 95\% Cl: $1.069-3.720$ for item 10 to OR: $3.296,95 \%$ Cl: $1.071-10.140$ for item 3$)$. The occurrence of falls without severe injury was not selected by the multinomial logistic regression technique as predictive of dependence in any specific ADL.

\subsection{AADL, IADL and BADL dependence secondary results}

Like in the previous analysis, these results were adjusted for the selected confounders computed by binomial logistic regression (Table 3). For AADL and more demanding IADL, adjustments were made for health conditions, aerobic endurance, multidimensional balance and lean body mass index, $p<.05$. For less demanding IADL and BADL, adjustments were made for health conditions, multidimensional balance, strength, flexibility and body fat mass, $p<.05$. Results were also adjusted for physical activity in three ADL (items $3,8,12), p<.05$. The analyses of these secondary results showed that all these confounders were predictive of ADL performance, in which a poorer outcome was associated with an increased likelihood of dependence $(p<.05)$.

\section{4 | DISCUSSION}

The present study showed that FoF explains overall physical dependence and represents a constraint on the performance of most basic, instrumental and advanced ADL. On the other hand, the occurrence of falls seems to explain less the ADL dependence than fear, even with severe injuries and overcoming the acute phase. This, whatever is the chronological age, health conditions, physical fitness, body composition or physical activity.

Increased FoF augmented up to 3 times the likelihood of a person achieves the condition of "moderate function" and up to 7 times the likelihood for the condition "low function," even in the absence of falls and independently of fall-related injuries occurrence. Particularly, as long as the FoF score increased, the likelihood of dependence in BADL, IADL and AADL increased, being 2-3 times higher than in cases of no fear. These findings are in accordance with other studies reporting the restriction of ADL due to FoF (Choi \& Ko, 2015; Lavedan et al., 2018). On the other way, the occurrence of falls causing light or no injuries was not a significant predictor of ADL dependence, while fall-related severe injuries revealed to increase the likelihood of a person to have moderate ( 3 times) or even low (6 times) physical function. In addition, severe injuries were shown to increase the likelihood of dependence by 3-4 times in more demanding IADL, such as lifting and carrying heavy objects. These results are important to quantify the role of falls and fall-related injuries in the ADL performance of community-dwelling older persons, complementing previous observations that reported that a decline in overall ADL was associated with the severity of injuries (Gill, Allore, Gahbauer, \& Murphy, 2012).

The occurrence of falls and injuries has been associated with FoF (Lavedan et al., 2018). On this way and complementarily, the findings of Lavedan et al. (2018), in the present study, were observed that the occurrence of falls with or without injuries was associated with a greater FoF and that a poor health conditions, physical activity, physical fitness and body composition were also associated with a greater FoF, even in the absence of falls. In fact, fear may also be caused by fragility (Chu et al., 2011; Denkinger et al., 2015; Hoang et al., 2017) and obesity (Neri et al., 2017). Therefore, the self-perception of fragility might inhibit the performance of ADL and lead subjects to avoid activities that they can perform (Tinetti et al., 1990). However, in our study, the effects of health conditions and other confounder factors that may promote dependence in ADL performance were controlled by multinomial analysis or logistic regression analysis, meaning that the quantitative founds for risk of dependency described above concerns to FoF and for falls and injuries by themselves. These results suggest that, more than falls and injuries occurrence, a lack of self-confidence in performing ADL safely plays the main role in their performance restriction. Ruthig et al. (2007) have already found associations between FoF, negative emotions, decreased perceived control and activity restriction. This can be a problem because the restriction of activity due to FoF leads to further deterioration and dependence in ADL (Choi \& Ko, 2015; Deshpande et al., 2008).

Secondary results of the present study showed that the maintenance of independence in IADL and AADL, apart from being associated with fear and injuries by itself, was associated with health conditions, aerobic endurance, multidimensional balance and lean 
TABLE 2 Regression model explaining low and moderate physical function

\begin{tabular}{|c|c|c|c|}
\hline & Variables & Odds ratio & $95 \% \mathrm{Cl}$ \\
\hline \multirow{5}{*}{ Low physical function } & $\begin{array}{l}\text { Lean body mass index } \\
\left(\mathrm{kg} / \mathrm{m}^{2}\right)\end{array}$ & 0.747 & $0.631-0.883$ \\
\hline & Aerobic endurance $(\mathrm{m})$ & 0.989 & $0.983-0.994$ \\
\hline & $\begin{array}{l}\text { Multidimensional } \\
\text { balance (point) }\end{array}$ & 0.901 & $0.828-0.979$ \\
\hline & $\mathrm{FES}+{ }^{\mathrm{a}}$ & 6.616 & $2.444-17.912$ \\
\hline & Injury+ & 5.548 & $1.521-20.240$ \\
\hline \multirow[t]{5}{*}{ Moderate physical function } & Health conditions ( $n$ ) & 1.172 & $1.030-1.332$ \\
\hline & $\begin{array}{l}\text { Lean body mass index } \\
\left(\mathrm{kg} / \mathrm{m}^{2}\right)\end{array}$ & 0.752 & $0.669-0.845$ \\
\hline & $\mathrm{FES} \pm^{\mathrm{a}}$ & 2.079 & $1.086-3.979$ \\
\hline & $\mathrm{FES}+^{\mathrm{a}}$ & 2.710 & $1.217-6.035$ \\
\hline & Injury+ & 2.500 & $1.050-5.950$ \\
\hline
\end{tabular}

Abbreviations: $\mathrm{Cl}$, confidence interval; FES, fear of falling assessed by Falls Efficacy Scale.

${ }^{\mathrm{a}} \mathrm{FES}-$, as reference category.

body mass index. Regarding less demanding ADL, besides health status and multidimensional balance, muscle strength, flexibility and low-fat body mass also seem to be determinants indicators of dependence. These observations, despite being based on cross-sectional data, reinforce our main results once they are in accordance with previous experimental studies, which reported a significant correlation between the decrease in ADL dependence and the increment of muscle strength, flexibility and balance induced by exercise programs (Carral \& Pérez, 2007; Sato et al., 2011). Our findings are also in accordance with a study (den Ouden, Schuurmans, Arts, \& van der Schouw, 2016), which found a significant association between lung function and higher leg strength, with a higher probability of being independent in ADL.

Some researches show that physical activity promotes quality of life in elderly people, in particular health status, physical fitness and independent functioning (Paterson \& Warburton, 2010; Pereira et al., 2016). In the present study, it was observed that physical activity performance was predictive of three ADL ability (climbing up and down a flight of stairs, walking 1 mile and doing strenuous activities), regardless of health, physical fitness level, body composition, FoF or the occurrence of severe injuries, although physical activity performance has also shown to do not explain significantly general physical function. Furthermore, if on one hand physical activity performance was found to be associated with high physical fitness and favourable body composition, on the other hand, it was significantly associated with low scores of FoF, regardless of health, physical fitness level, body composition or the occurrence of severe injuries. These new findings complement the knowledge about the role of physical activity in the maintenance of independence in community-dwelling older adults. Indeed, our findings report the direct role of physical activity on specific ADLs' ability and the indirect role of physical activity on physical independence mediated by its association with variables that explain general and single ADLs' functioning, particularly FoF. Thus, the main findings of the present study suggest that interventions targeting ADL independence shall focus self-confidence of individuals through the successful accomplishment of functional exercises that mimic ADL and not just favour exercises targeting body composition and fitness improvement. This is in line with the findings of Auais et al. $(2016$, 2018), who found that FoF was associated with an increased risk of developing mobility disability and poor physical performance.

We also observed that older old adults were more dependent than younger old adults, as well as that woman were more dependent than men, as expected and in accordance with other studies (Rubio et al., 2009). However, when adjusted for health status, physical fitness, and body composition, age and gender were not predictors of dependence in persons with similar fall-related injuries or FoF. Previous studies also reported that age and gender lose their predictive ability of physical independence when adjusted for health status, physical fitness and body composition (Pereira et al., 2016). Moreover, present study reinforces the idea suggested by others (Fujiwara et al., 2008; Himes \& Reynolds, 2012; Tinetti \& Kumar, 2010) that the risk factors for ADL dependence are common for falls and fall-related injuries, especially health conditions, physical fitness, body composition, physical activity and FoF. Thus, there may be common preventive strategies for both these negative outcomes.

Statistical interactions between health conditions, habitual physical activity, physical fitness or body composition with FoF and 
FIGURE 3 Dependence in advanced (AADLs), instrumental (IADLs) and basic activities of daily living (BADLs) significantly associated with the occurrence of severe fall-related injuries and with fear of falling (results computed in opposition to independent category). The figure illustrates the increase in the likelihood of a person to be dependent (cannot do or can do with difficulty or help)-with regard to the performance of each $A D L-a s s o c i a t e d$ with the occurrence of severe fall-related injuries (Injury+) and with increased fear of falling (FES \pm or FES+). Only items with significant associations are shown. The reference categories were as follows: no fall or fall without injury or with light injury (Injury-); and no fear of falling (FES-: score 0-1). Item 3, "Walk outside (1 or 2 blocks)"; Item 8, "Walk 1/2 mile (6-7 blocks)"; Item 9, "Walk 1 mile (12-14 blocks)"; Item 10, "Lift and carry $25 \mathrm{lb}$ (medium to large suitcase)"; Item 11, "Do heavy household activities-like scrubbing floors, vacuuming, raking leaves"; Item 12, "Do strenuous activities-like hiking, digging in the garden, moving heavy objects, cycling, aerobic dance activities, strenuous calisthenics." $\mathrm{Cl}$, confidence interval

\section{Dependence odds ratio (95\% CI)}

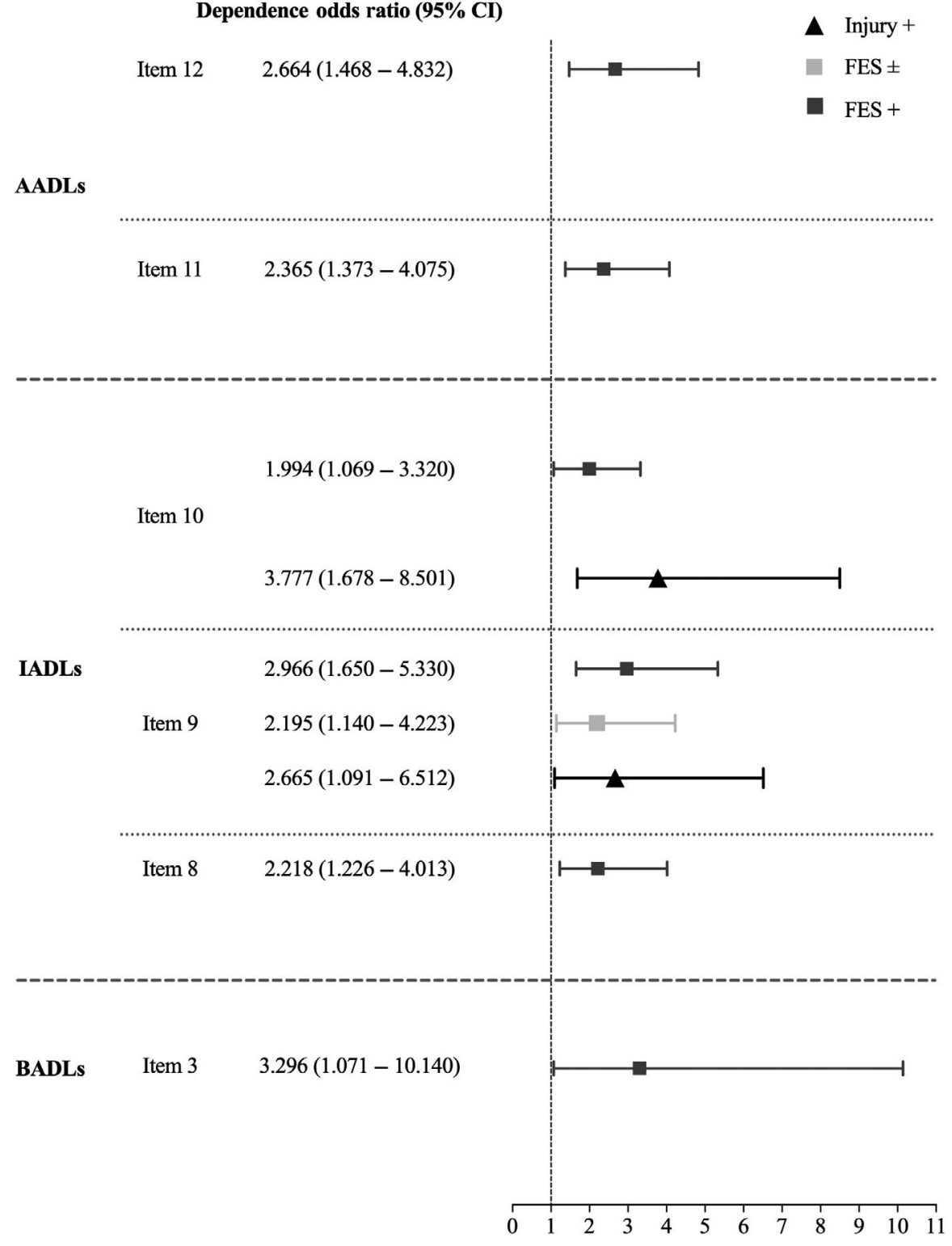

falls and injuries occurrence, which have not been reported in previous studies, seem to play an essential role on the association between FoF and falls and injuries with the risk of ADL dependence in community-dwelling older adults. Thus, the interplay between confounders with the FoF and falls and injuries, as significant determinants of risk of ADL dependence, might be more complex than the chronological and gender pathways of the ageing process. The present study extends previous findings by examining the isolated association between risk for ADL dependence, measured through $\mathrm{CPF}$, and the FoF or falls and injuries by controlling relationships for significant confounders. Therefore, nurses and other healthcare professionals should include the FoF in clinical evaluations once FoF showed a stronger relationship with physical dependence than previous falls and fall-related injuries. Hence, interventions to reduce the risk of physical dependence, besides focusing on health conditions, physical fitness and body composition, should consider the management of FoF.

\section{1 | Study limitations}

The limitations of this study include the relative ability of crosssectional studies to establish cause-effect relationships; as this is a cross-sectional data set, reverse causality cannot be ruled out. Besides, and as expected, community-dwelling participants revealed low scores of FoF, and only a few participants showed dependence in performing less demanding ADL (particularly BADL), diminishing the statistical power of the analysis, particularly that focused on these activities. The physical activity, CPF scale and falls occurrence were assessed through questionnaire, and as many participants could not read or write, it could be recognised as a limitation; however, it was minimised by the use of an interviewer who completed the questionnaire for each participant and the inclusion of criteria for the absence of cognitive impairment. This strategy also contributed to avoiding missing falls and fall-related injuries since fall circumstances were systematically checked along with the interview, as well as to control 
TABLE 3 Regression models explaining AADL, IADL and BADL dependence

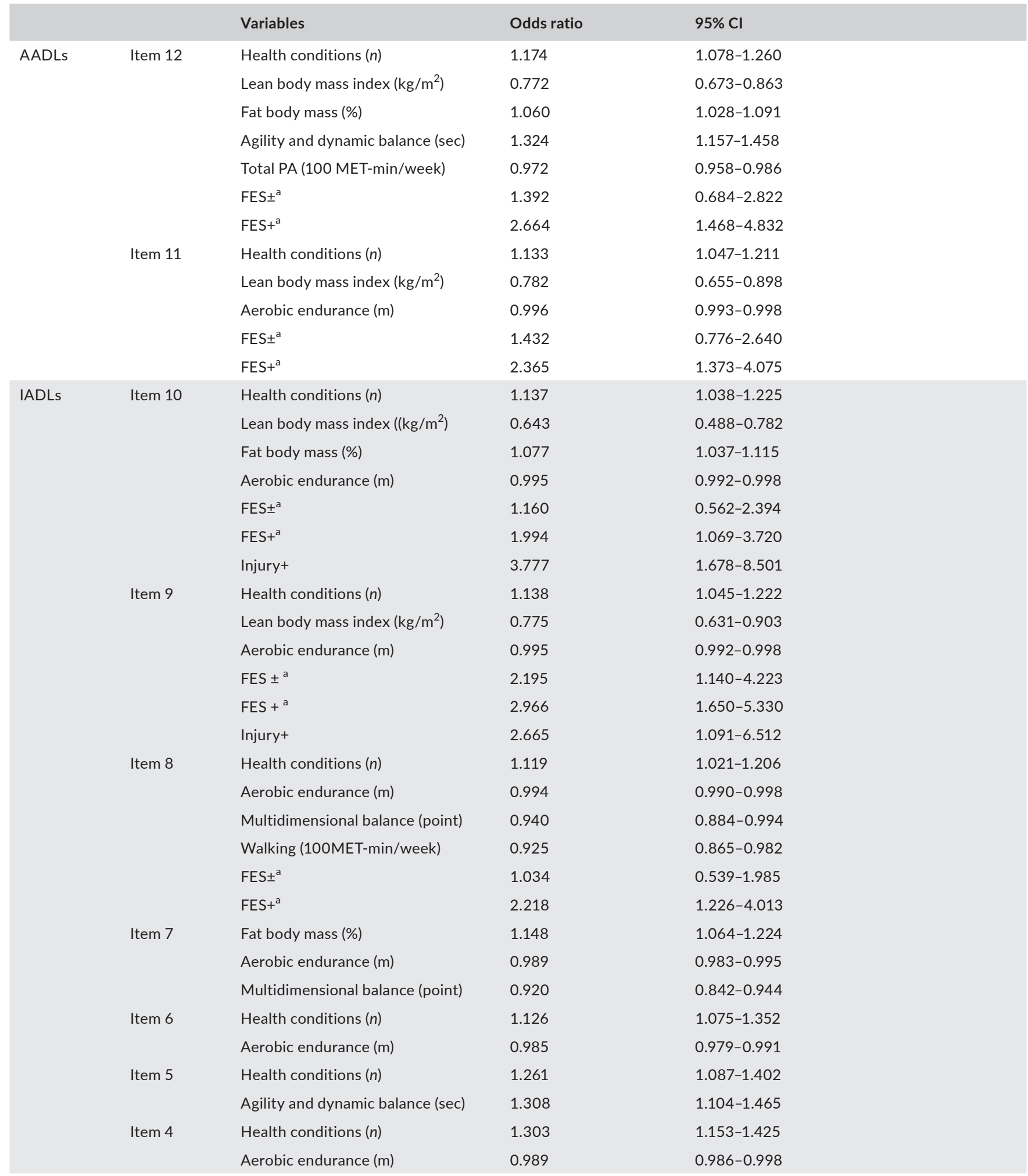


TABLE 3 (Continued)

\begin{tabular}{|c|c|c|c|c|}
\hline & & Variables & Odds ratio & $95 \% \mathrm{Cl}$ \\
\hline \multirow[t]{9}{*}{ BADLs } & \multirow[t]{5}{*}{ Item 3} & Fat body mass (\%) & 1.083 & $1.034-1.130$ \\
\hline & & Aerobic endurance (m) & 0.989 & $0.984-0.994$ \\
\hline & & Exercise (hr/week) & 0.208 & $0.159-0.491$ \\
\hline & & $\mathrm{FES} \pm^{\mathrm{a}}$ & 3.661 & $0.938-14.278$ \\
\hline & & $\mathrm{FES}+{ }^{\mathrm{a}}$ & 3.296 & $1.071-10.140$ \\
\hline & \multirow[t]{3}{*}{ Item 2} & Health conditions ( $n$ ) & 1.331 & $1.075-1.516$ \\
\hline & & Fat body mass (\%) & 1.349 & $1.025-1.565$ \\
\hline & & Upper body strength (rep) & 0.564 & $0.103-0.912$ \\
\hline & Item 1 & Upper body flexibility (cm) & 0.902 & $0.814-0.984$ \\
\hline
\end{tabular}

Abbreviations: AADL's, advanced activities of daily living; BADLs, basic activities of daily living; $\mathrm{Cl}$, confidence interval; FES, fear of falling assessed by Falls Efficacy Scale; IADLs, instrumental activities of daily living.

aFES-as reference category. Item 1, "Take care of own personal needs, such as dressing yourself"; Item 2, "Bathe yourself, using tub or shower"; Item 3, "Walk outside (1 or 2 blocks)"; Item 4, "Do light household chores, such as cooking, dusting, washing dishes, sweeping a walkway"; Item 5, "Climb up and down a flight of stairs"; Item 6, "Do own shopping and errands (walk approx 3-4 blks; 400 yds)"; Item 7, "Lift and carry 10 pounds (bag of groceries)"; Item 8, "Walk 1/2 mile (6-7 blocks)"; Item 9, "Walk 1 mile (12-14 blocks)"; Item 10, "Lift and carry 25 lb (medium to large suitcase)"; Item 11, "Do heavy household activities-like scrubbing floors, vacuuming, raking leaves"; Item 12, "Do strenuous activities-like hiking, digging in the garden, moving heavy objects, cycling, aerobic dance activities, strenuous calisthenics."

eventual overestimation of physical activity. These limitations prevent the generalisation of present finds to all populations. Despite the limitations, present study findings may have implications for the future development of assessment guidelines for older adults, allowing nursing and other healthcare professionals to design more successful interventions to prevent further dependence. Future controlled trial studies should investigate the effect of interventions targeting physical dependence considering the potential mediators of FoF and falls and injuries and should also examine causality by testing the impact of reducing FoF and falls and injuries occurrence on dependence.

\section{5 | CONCLUSION}

In conclusion, these new findings suggest that the FoF is significantly associated with the risk of physical dependence in older

\section{Implications for practice}

- These study main results indicate that physical dependence in community-dwelling older adults may be directly influenced by the FoF, independently of age, health conditions, physical fitness, body composition and physical activity.

- The FoF showed to be strongly related to physical dependence, restricting the performance of the main ADL.

- Healthcare professionals are advised to include the FoF in clinical evaluations and to design interventions to reduce the risk of physical dependence considering the management of FoF. adults, independently of the effect of chronological age, health conditions, physical fitness, body composition and physical activity. Overall, the FoF proved to be a greater predictor than falls and related injuries for physical dependence, restricting the performance of AADL, IADL and BADL. Regarding these risk factors, $A D L$ dependence in community-dwelling older adults seems to occur mainly in advanced and more demanding instrumental ADL than basic ADL. Nurses and healthcare professionals shall consider these findings on the development of interventions to prevent physical dependence and include FoF in clinical evaluations of older adults living in the community.

\section{ACKNOWLEDGEMENTS}

The authors would like to thank all the participants who have collaborated in this study in an unselfish manner.

\section{CONFLICT OF INTEREST}

The authors declare that they have no conflict of interest.

\section{AUTHOR CONTRIBUTIONS}

Study design: CP; Supervision and data collection: CP, FB; Data analysis and writing manuscript (with support from AR and FM): CP, PC, $J B$; all authors reviewed and provided input into the final version of the manuscript.

\section{ORCID}

Catarina Pereira (iD https://orcid.org/0000-0001-8111-1455

Jorge Bravo (iD https://orcid.org/0000-0002-9009-5693

Armando Raimundo (iD https://orcid.org/0000-0001-9753-8381

Pablo Tomas-Carus (iD https://orcid.org/0000-0001-7780-3942

Felismina Mendes (iD https://orcid.org/0000-0001-9518-2289

Fátima Baptista (iD https://orcid.org/0000-0002-0214-5401 


\section{REFERENCES}

Auais, M., Alvarado, B. E., Curcio, C. L., Garcia, A., Ylli, A., \& Deshpande, N. (2016). Fear of falling as a risk factor of mobility disability in older people at five diverse sites of the IMIAS study. Archives of Gerontology and Geriatrics, 66, 147-153. https://doi.org/10.1016/j. archger.2016.05.012

Auais, M., French, S., Alvarado, B., Pirkle, C., Belanger, E., \& Guralnik, J. (2018). Fear of falling predicts incidence of functional disability 2 years later: a perspective from an international cohort study. The Journals of Gerontology Series A Biological Sciences and Medical Sciences, 73(9), 1212-1215. https://doi.org/10.1093/gerona/glx237

Bowling, A., \& Iliffe, S. (2006). Which model of successful ageing should be used? Baseline findings from a British longitudinal survey of ageing. Age and Ageing, 35(6), 607-614. https://doi.org/10.1093/ageing/ afl100

Carral, J. C., \& Pérez, C. A. (2007). Effects of high-intensity combined training on women over 65. Gerontology, 53(6), 340-346. https://doi. org/10.1159/000104098

Chodzko-Zajko, W., Proctor, D., Fiatarone Singh, M., Minson, C., Nigg, C., Salem, G., \& Skinner, J. (2009). American College of Sports Medicine Position Stand. Exercise and physical activity for older adults. Medicine \& Science in Sports \& Exercise, 41(7), 1510-1530. https://doi. org/10.1249/MSS.0b013e3181a0c95c

Choi, K., \& Ko, Y. (2015). Characteristics associated with fear of falling and activity restriction in South Korean older adults. Journal of Aging and Health, 27(6), 1066-1083. https://doi.org/10.1177/0898264315 573519

Chu, C.-L., Liang, C.-K., Chow, P. C., Lin, Y.-T., Tang, K.-Y., Chou, M.-Y., ... Pan, C.-C. (2011). Fear of falling (FF): Psychosocial and physical factors among institutionalized older Chinese men in Taiwan. Archives of Gerontology and Geriatrics, 53(2), 232-236. https://doi.org/10.1016/j. archger.2010.12.018

Craig, C. L., Marshall, A. L., Sjöström, M., Bauman, A. E., Booth, M. L., Ainsworth, B. E., ... Oja, P. (2003). International physical activity questionnaire: 12 -country reliability and validity. Medicine \& Science in Sports \& Exercise, 35(8), 1381-1395. https://doi.org/10.1249/01. MSS.0000078924.61453.FB

Curcio, C. L., Gomez, F., \& Reyes-Ortiz, C. A. (2009). Activity restriction related to fear of falling among older people in the Colombian Andes mountains: Are functional or psychosocial risk factors more important? Journal of Aging and Health, 21(3), 460-479. https://doi. org/10.1177/0898264308329024

Dean, A., Sullivan, K., \& Soe, M. (2014). OpenEpi: Open source epidemiologic statistics for public health (version 2.3.1.). Retrieved from www. OpenEpi.com

den Ouden, M. E., Schuurmans, M. J., Arts, I. E., \& van der Schouw, Y. T. (2016). Association between physical performance characteristics and independence in activities of daily living in middle-aged and elderly men. Geriatrics \& Gerontology International, 13(2), 274-280. https://doi.org/10.1111/j.1447-0594.2012.00890.x

Denkinger, M., Lukas, A., Nikolaus, T., \& Hauer, K. (2015). Factors associated with fear of falling and associated activity restriction in community-dwelling older adults: A systematic review. The American Journal of Geriatric Psychiatry, 23(1), 72-86. https://doi.org/10.1016/j. jagp.2014.03.002

Deshpande, N., Metter, E. J., Bandinelli, S., Lauretani, F., Windham, B. G., \& Ferrucci, L. (2008). Psychological, physical, and sensory correlates of fear of falling and consequent activity restriction in the elderly: The InCHIANTI study. American Journal of Physical Medicine \& Rehabilitation/Association of Academic Physiatrists, 87(5), 354-362. https://doi.org/10.1097/PHM.0b013e31815e6e9b

Folstein, M. F., Folstein, S. E., \& McHugh, P. R. (1975). "Mini-mental state". A practical method for grading the cognitive state of patients for the clinician. Journal of Psychiatric Research, 12(3), 189-198. http://doi. org/10.0022-3956(75)90026-6
Fujiwara, Y., Yoshida, H., Amano, H., Fukaya, T., Liang, J., Uchida, H., \& Shinkai, S. (2008). Predictors of improvement or decline in instrumental activities of daily living among community-dwelling older Japanese. Gerontology, 54(6), 373-380. https://doi. org/10.1159/000151221

Gill, T. M., Allore, H. G., Gahbauer, E. A., \& Murphy, T. E. (2012). Change in disability after hospitalization or restricted activity in older persons. Journal of the American Medical Association, 304(17), 1919-1928. https://doi.org/10.1001/jama.2010.1568

Gómez, F., Wu, Y. Y., Auais, M., Vafaei, A., \& Zunzunegui, M.-V. (2017). A simple algorithm to predict falls in primary care patients aged 65 to 74 years: The International Mobility in Aging Study. Journal of the American Medical Directors Association, 18(9), 774-779.

Himes, C. L., \& Reynolds, S. L. (2012). Effect of obesity on falls, injury, and disability. Journal of the American Geriatrics Society, 60(1), 124129. https://doi.org/10.1111/j.1532-5415.2011.03767.x

Hoang, O. T., Jullamate, P., Piphatvanitcha, N., \& Rosenberg, E. (2017). Factors related to fear of falling among community-dwelling older adults. Journal of Clinical Nursing, 26(1-2), 68-76. https://doi. org/10.1111/jocn.13337

INE (2019). Census 2011: Resident population (No.) by place of residence, sex and age group (by life cycles). Retrieved from http://www.ine.pt

Lavedan, A., Viladrosa, M., Jurschik, P., Botigue, T., Nuin, C., Masot, O., \& Lavedan, R. (2018). Correction: Fear of falling in community-dwelling older adults: A cause of falls, a consequence, or both? PLOS ONE, 13(5), e0197792. https://doi.org/10.1371/journal.pone.0197792

Neri, S. G. R., Gadelha, A. B., de David, A. C., Ferreira, A. P., Safons, M. P., Tiedemann, A., \& Lima, R. M. (2017). The association between body adiposity measures, postural balance, fear of falling, and fall risk in older community-dwelling women. Journal of Geriatric Physical Therapy, 42(3), E94-E100. https://doi.org/10.1519/JPT.00000 00000000165

Paterson, D. H., \& Warburton, D. E. (2010). Physical activity and functional limitations in older adults: A systematic review related to Canada's Physical Activity Guidelines. International Journal of Behavioral Nutrition and Physical Activity, 7, 38. https://doi.org/10.1186/1479-5868-7-38

Pereira, C., Baptista, F., \& Cruz-Ferreira, A. (2016). Role of physical activity, physical fitness, and chronic health conditions on the physical independence of community-dwelling older adults over a 5-year period. Archives of Gerontology and Geriatrics, 65, 45-53. https://doi. org/10.1016/j.archger.2016.02.004

Pereira, C., Baptista, F., \& Infante, P. (2014). Role of physical activity in the occurrence of falls and fall-related injuries in community-dwelling adults over 50 years old. Disability and Rehabilitation, 36(2), 117124. https://doi.org/10.3109/09638288.2013.782355

Pluijm, S. M., Smit, J. H., Tromp, E. A., Stel, V. S., Deeg, D. J., Bouter, L. M., \& Lips, P. (2006). A risk profile for identifying community-dwelling elderly with a high risk of recurrent falling: Results of a 3-year prospective study. Osteoporosis International, 17(3), 417-425. https ://doi.org/10.1007/s00198-005-0002-0

Rikli, R., \& Jones, C. (1998). The reliability and validity of a 6-minute walk test as a measure of physical endurance in older adults. Journal of Aging and Physical Activity, 6, 363-375. https://doi.org/10.1123/ japa.6.4.363

Rikli, R., \& Jones, C. (2013). Development and validation of criterion-referenced clinically relevant fitness standards for maintaining physical independence in later years. Gerontologist, 53(2), 255-267. https:// doi.org/10.1093/geront/gns071

Rose, D. J., Lucchese, N., \& Wiersma, L. D. (2006). Development of a multidimensional balance scale for use with functionally independent older adults. Archives of Physical Medicine and Rehabilitation, 87(11), 1478-1485. https://doi.org/10.1016/j.apmr.2006.07.263

Rubio, E., Lazaro, A., \& Sanchez-Sanchez, A. (2009). Social participation and independence in activities of daily living: A cross sectional study. BMC Geriatrics, 9, 26. https://doi.org/10.1186/1471-2318-9-26 
Ruthig, J. C., Chipperfield, J. G., Newall, N. E., Perry, R. P., \& Hall, N. C. (2007). Detrimental effects of falling on health and well-being in later life: The mediating roles of perceived control and optimism. Journal of Health Psychology, 12(2), 231-248. https://doi.org/10.1177/13591 05307074250

Sato, D., Kaneda, K., Wakabayashi, H., Shimoyama, Y., Baba, Y., \& Nomura, T. (2011). Comparison of once and twice weekly water exercise on various bodily functions in community-dwelling frail elderly requiring nursing care. Archives of Gerontology and Geriatrics, 52(3), 331-335. https://doi.org/10.1016/j.archger.2010.05.002

Scheffer, A. C., Schuurmans, M. J., Van Dijk, N., Van Der Hooft, T., \& De Rooij, S. E. (2008). Fear of falling: Measurement strategy, prevalence, risk factors and consequences among older persons. Age and Ageing, 37(1), 19-24. https://doi.org/10.1093/ageing/afm169

Tinetti, M., \& Kumar, C. (2010). The patient who falls: "It's always a tradeoff". Journal of the American Medical Association, 303(3), 258-266. https://doi.org/10.1001/jama.2009.2024

Tinetti, M., Mendes de Leon, C., Doucette, J., \& Baker, D. (1994). Fear of falling and fall-related efficacy in relationship to functioning among community-living elders. Journal of Gerontology, 49(3), M140-M147. https://doi.org/10.1093/geronj/49.3.M140
Tinetti, M., Richman, D., \& Powell, L. (1990). Falls efficacy as a measure of fear of falling. Journal of Gerontology, 45(6), P239- P243. https://doi. org/10.1093/geronj/45.6.P239

Zaluska, W., Malecka, T., Mozul, S., \& Ksiazek, A. (2004). Whole body versus segmental bioimpedance measurements (BIS) of electrical resistance (Re) and extracellular volume (ECV) for assessment of dry weight in end-stage renal patients treated by hemodialysis. Przeglad Lekarski, 61(2), 70-73.

How to cite this article: Pereira C, Bravo J, Raimundo A, Tomas-Carus P, Mendes F, Baptista F. Risk for physical dependence in community-dwelling older adults: The role of fear of falling, falls and fall-related injuries. Int J Older People Nurs. 2020;00:e12310. https://doi.org/10.1111/opn.12310 